\title{
Disruption of the Gut Microbiota Attenuates Epithelial Ovarian Cancer Sensitivity to Cisplatin Therapy
}

\section{Laura Chambers}

Cleveland Clinic

\section{Emily Esakov}

Cleveland Clinic Foundation: Cleveland Clinic https://orcid.org/0000-0002-5049-2096

\section{Chad Braley}

Cleveland Clinic

Lexie Trestan

Cleveland Clinic

\section{Zahraa Alali}

Cleveland Clinic

\section{Peter Bazeley}

Cleveland Clinic

\section{Defne Bayik}

Cleveland Clinic

Justin D Lathia

Cleveland Clinic

Naseer Sangwan

Cleveland Clinic

Amy S Joehlin-Price

Cleveland Clinic

Mohammed Dwidar

Cleveland Clinic

\section{Adeline Hajjar}

Cleveland Clinic

Philip P Ahern

Cleveland Clinic

\section{Jan Claesen}

Cleveland Clinic

\section{Peter Rose}

Cleveland Clinic

\section{Roberto Vargas}

Cleveland Clinic

\section{Chad Michener}

Cleveland Clinic

Ofer Reizes ( $\nabla$ reizeso@ccf.org )

Department of Cardiovascular and Metabolic Sciences, Center for Microbiome and Human Health, Lerner Research Institute, Cleveland Clinic, Cleveland, $\mathrm{OH}$ https://orcid.org/0000-0003-0075-6871 


\section{Research}

Keywords: Epithelial Ovarian Cancer (EOC), intraperitoneally injected, metronidazole

Posted Date: September 25th, 2020

DOI: https://doi.org/10.21203/rs.3.rs-80626/v1

License: (c) (i) This work is licensed under a Creative Commons Attribution 4.0 International License. Read Full License 


\section{Abstract}

Background: Epithelial Ovarian Cancer (EOC) is the second most common gynecologic malignancy in the United States, but the leading cause of gynecologic cancer death. Despite many achieving remission with first-line therapy, up to $80 \%$ of patients will recur and require additional treatment. Antibiotic therapy is frequently used during cancer treatments for both prophylaxis and treatment of infections, although this profoundly impacts the gut microbiome. Multiple studies suggest that an unperturbed gut microbiome may provide a protective microenvironment, and disruption may be permissive to tumor growth and chemotherapy resistance, including platinum agents.

Experimental Design: We assessed whether antibiotic therapy would impact growth of EOC and sensitivity to cisplatin in murine models. Immune competent or compromised mice were given either metronidazole, ampicillin, vancomycin, and neomycin (ABX) containing or control water for two weeks before being intraperitoneally injected with murine ID8 or ID8-VEGF EOC cells. Tumors were monitored and cisplatin therapy was administered weekly until endpoint. Stool was collected throughout the study to asses for microbial population effects over time.

Results: Both immune competent and immune compromised ID8 and ID8 VEGF tumor-bearing mice demonstrated a decreased response to cisplatin therapy in ABX treated groups with an increase in overall tumor burden. RNAseq analysis showed enrichment of multiple cell proliferation and stem cell pathways, and stem cell genes SOX2, WNT and PAX2. The self-renewal of ABX treated tumor cells was also increased.

Conclusion: Collectively, these studies indicate an intact microbiome provides a tumor suppressive microenvironment and enhances sensitivity to cisplatin.

\section{Introduction}

Epithelial ovarian, peritoneal and fallopian tube carcinomas (EOC) are a leading cause of gynecologic cancer related death (1). Following EOC diagnosis, patients are treated with a combination of cytoreductive surgery and platinumtaxane based chemotherapy (2-8). Unfortunately, despite many women achieving remission with first-line therapy, up to $80 \%$ of patients will recur and require additional treatment (2-8). After disease recurrence, the interval from last treatment with platinum chemotherapy has important therapeutic and prognostic implications (9). Patients with platinum resistant EOC have fewer treatment options, reduced response rates to chemotherapy and poor prognosis compared to those with platinum sensitive disease (9). There is a significant unmet need to further understand the mechanisms of platinum resistance in EOC and to advance current therapeutic options for these patients.

The gut microbiome has many roles in maintenance of human health, and has been increasingly linked with many disease states, including cancer (10-18). Recent evidence suggests that the gut microbiome may modulate responses to cancer treatment, including traditional chemotherapy and immunotherapy (10-19). In a study by Routy et al, resistance to immune checkpoint inhibitors was linked to abnormal gut microbiome composition following treatment with antibiotics (18). Similarly, lida et al. evaluated the role of the gut microbiome upon platinum chemotherapy response in mice with lymphoma, colon cancer and melanoma tumors (14). In mice treated with antibiotics that induce disruption of the microbiome, the impact of oxaliplatin treatment was attenuated, with decreased tumor regression and worse survival, compared to non-antibiotic control animals.

Antibiotic therapy is frequently used during cancer treatments for both prophylaxis and treatment of infections. Studies have demonstrated that receipt of antibiotics during both systemic chemotherapy and immunotherapy negatively impacts oncologic outcomes. In a study of patients with relapsed lymphoma and leukemia receiving either cisplatin or cyclophosphamide on two clinical trials, those that received antibiotics against gram positive bacteria during platinum 
chemotherapy had a significant reduction in overall treatment response, time to recurrence and overall survival (17). Among patients with epithelial ovarian cancer, studies have demonstrated that treatment for surgical site infection following primary cytoreductive surgery is associated with worse survival (20). In a recent retrospective analysis, EOC patients who received antibiotics, primarily against gram positive bacteria, during primary platinum chemotherapy had reduced overall (> 17 months) and progression free survival (>24 months) compared to patients who received no antibiotic intervention (21). These studies suggest that an unperturbed gut microbiome may provide a protective microenvironment, and disruption may be permissive to tumor growth and chemotherapy resistance, including platinum agents. Here, we investigated the impact of antibiotic mediated disruption of the gut microbiome on EOC tumor growth and platinum chemotherapy response in pre-clinical models of EOC.

\section{Results}

\section{EOC tumors exhibit accelerated growth and attenuated sensitivity to cisplatin in antibiotic treated mice.}

We tested the hypothesis that an antibiotic driven disruption of the microbiome impacts tumor development and chemotherapy sensitivity in EOC. To this end, the following study paradigm (Fig. 1A) was implemented wherein C57 $\mathrm{BL} / 6 \mathrm{~J}(\mathrm{BL} / 6)$ female mice at 6 weeks of age were provided water or antibiotic containing water (ABX; ampicillin, neomycin, metronidazole, and vancomycin), which was sustained for the duration of the study. After 2 weeks, mice were injected intraperitoneally (IP) with murine EOC lines ID8 or ID8-VEGF, syngeneic with BL/6 mice, and tumor growth was monitored by transabdominal ultrasound (TAUS) weekly for the course of the study (22). ID8 and ID8-VEGF cell lines were utilized as they are highly characterized and closely recapitulate human ovarian cancer progression. The ID8-VEGF cell line is slightly more severe causing an increase in angiogenesis and ascites development $(23,24)$. At 2 weeks post tumor cell injection, mice were injected IP with cisplatin or vehicle weekly for the remainder of the study. Mice met study endpoint at tumor burden of $>150 \mathrm{~mm}^{3}$ or humane endpoint, including debilitating ascites development. Upon necropsy, EOC tumor phenotype was confirmed through histological assessment of H\&E stained tissue sections as well as benign adjacent omentum (Supplemental Fig. 1A). Our findings indicate that EOC tumor growth is significantly increased by $A B X$ treated mice compared to controls. In the presence of ABX, cisplatin therapy had attenuated efficacy compared to in the control treated mice indicating development of cisplatin resistance (Fig. 1B, D). As expected from the tumor burden, median survival was decreased in the ABX treatment groups compared to controls. The ID8 cohort exhibited a median survival of 66 and 64 days in the ABX placebo and cisplatin groups compared to 68.5 and 84 days in the $\mathrm{H}_{2} \mathrm{O}$ placebo and cisplatin groups, respectively (Fig. 1C). This result was paralleled in the ID8-VEGF cohort of mice with a median survival of 39 and 34 days in the ABX placebo and cisplatin groups compared to 42 and 53 days in the $\mathrm{H}_{2} \mathrm{O}$ placebo and cisplatin groups respectively (Fig. 1E). The ID8-VEGF mice reached the endpoint earlier than the ID8 cohort secondary to large volume ascites development.

\section{Limited impact on immune populations in ascites of EOC by broad spectrum antibiotics.}

The majority of EOC patients present with advanced disease (stage III or stage IV) that may include ascites $(25,26)$. Although the underlying mechanisms of ascites development are largely unknown, previous studies have reported that immune cells such as Th17 T cells, NK cells and macrophages found in peritoneal ascites may play a key role in tumor cell invasiveness and growth (27-30). To this end, we analyzed the immune cell populations within the peritoneal ascites fluid through flow cytometry. Our flow cytometry evaluation of immune cell populations within the peritoneal ascites was designed to focus on both myeloid and lymphoid populations (see Supplemental Fig. 2 for antibody panels and gating strategies). Our findings indicated no significant difference in myeloid (Supplemental Fig. 3A) or lymphoid (Supplemental Fig. 3B) cell populations following ABX therapy in the presence or absence of cisplatin therapy in the BL/6 ID8-VEGF cohort ascites collected at endpoint. 
Disruption of the immune system does not significantly impact the accelerated EOC tumor growth or reduced cisplatin sensitivity in antibiotic treated mice.

To further assess whether the observed augmentation of tumor phenotype and cisplatin resistance following $A B X$ treatment is dependent on an intact immune system, the same study paradigm was utilized in NOD.Cg-Prkdc $<$ scid $>$ $\| 2 \mathrm{rg}<\mathrm{tm} 1 \mathrm{Wjl}>\mathrm{SzJ}$ (NSG) immuno-deficient mice. The NSG cohort displayed more significant impact of microbiome depletion through antibiotics on tumor growth as the $\mathrm{BL} / 6$ cohort, with no clinical benefit to the mice receiving cisplatin along with $A B X$ (Fig. 2A, C). The time to tumor progression was accelerated and the effect on median survival was even more dramatic with the ID8 cohort exhibiting a median survival of 41 and 45 days in the ABX placebo and cisplatin groups compared to 55 and 69 days in the $\mathrm{H}_{2} \mathrm{O}$ placebo and cisplatin groups respectively (Fig. 2B). A similar phenotype was observed in the ID8-VEGF cohort of NSG mice (Fig. 2D). As before, EOC tumors were confirmed via H\&E staining (Supplemental Fig. 1B). Additionally, the peritoneal ascites fluid at endpoint was analyzed by flow cytometry. NSG mice have immature T cells, DCs and macrophages, but functional neutrophils (31). NSG ID8-VEGF cohort ascites exhibited no significant alterations in myeloid (Supplemental Fig. $3 \mathrm{C}$ ) populations in ABX treated mice, regardless of cisplatin therapy compared to $\mathrm{H}_{2} \mathrm{O}$ controls.

\section{ABX treatment results in comparable disruptions of the gut microbiome in BL/6 and NSG EOC tumor bearing mice.}

The $A B X$ treatment regimen we applied was previously shown to be sufficient to deplete detectable commensal bacteria $(32,33)$. However, to define the response of the gut microbiome to broad spectrum antibiotic therapy, stool was collected at baseline, at 2 weeks, at tumor engraftment, at 5 weeks and at endpoint necropsy and then processed for 16S rRNA gene sequencing. The microbiome was analyzed from the following cohorts: BL/6 ID8 BL/6 ID8-VEGF, NSG ID8, and NSG ID8-VEGF.

C57 BL/6 cohort. In total, 2,801,471 and 2,411,554 high-quality and usable reads were obtained from fecal samples of 5 mice per treatment group in the ID8 and ID8-VEGF BL/ 6 cohorts from sequencing the 16S rRNA gene, with an average length of 210 base pairs (bps). There were 3,184 amplified sequence variants (ASVs) in all ID8 and ID8-VEGF BL/6 samples. Pairwise comparisons within both the ID8-VEGF and ID8 cohorts revealed statistically significant differences in alpha diversity between temporal collections, as analyzed by the Shannon diversity index (ANOVA $p<0.05$ ) (Supplemental Fig. 4A, B). Additionally, the Bray-Curtis dissimilarity based beta diversity comparisons between collection time points in the ID8-VEGF and ID8 cohorts were significant at $p=0.003$ and $p=0.001$ respectively (Supplemental Fig. 4C, D).

Antibiotic treated groups displayed significantly less diverse fecal microbial communities compared to control water treated groups regardless of cisplatin therapy. Specifically, the relative abundance of some Proteobacteria including Enterobacteriaceae and Parasutterella was increased in the antibiotic treated ID 8 groups while the abundance of Enterobacteriaceae was increased in the antibiotic treated ID8-VEGF groups (Fig. 3A, B). These were significantly increased by 2 weeks post antibiotic therapy in the mice being co treated with cisplatin, but took up to 5 weeks for the mice treated with placebo to obtain the same increase. Being Gram-negative, and mostly facultative anaerobes, the Proteobacteria are generally resistant to vancomycin which affects mainly Gram-positive bacteria and are also less susceptible to metronidazole which affects more the anaerobes (34). Furthermore, Enterobacteriaceae have multiple antibiotic resistance genes allowing for their survival post ABX therapy $(35,36)$. Increased abundance of Parasutterella has been associated with dysbiosis of the gut microbiome, but the mechanism has not been fully interrogated $(37,38)$.

NSG cohort. In total, 4,784,498 and 2,822,423 high-quality and usable reads were obtained from fecal samples of 5 mice per treatment group in the ID8 and ID8-VEGF NSG cohorts from sequencing the 16S rRNA gene, with an average length 210 bps. There were 6,484 ASVs in all ID8 and ID8-VEGF NSG samples. As with the BL/ 6 cohort, the pairwise 
comparisons revealed statistically significant (ANOVA, $p<0.05)$ patterns for alpha diversity (i.e. Shannon Index) between the collection time points in both the ID8-VEGF and ID8 cohorts (Supplemental Fig. 5A, B). Additionally, the Bray-Curtis dissimilarity based beta diversity between collection time points in the ID8-VEGF and ID8 cohorts were both significant at $p=0.001$ (Supplemental Fig. 5C, D).

Similar to the BL/ 6 cohort, the NSG cohort exhibited a marked relative increase in Enterobacteriaceae in the antibiotic treated ID8 and ID8-VEGF groups' fecal samples, increasing more quickly in the Cisplatin treated groups compared to the placebo as well. In addition, this cohort also saw a marked relative increase in Paenibacillus and Enterococcus (Fig. 4A, B).

\section{ABX treatment does not significantly alter ID8 or ID8-VEGF EOC tumor cell proliferation in vitro}

To determine if tumor growth was a direct effect of ABX interaction with the EOC ID8 and ID8-VEGF cell lines, we performed tissue culture analyses. ID8 and ID8-VEGF cell lines were co-cultured with various concentrations of ABX (metronidazole, vancomycin, ampicillin and neomycin) in the same respective ratios utilized in the murine studies Following 7 days culture, there were no appreciable differences in ABX treated cell lines compared to cell lines in normal media (Supplemental Fig. 6A, B). Additionally, there were no changes in the determined IC ${ }_{50}$ of cisplatin on ID8 of ID8-VEGF cells following ABX treatment when compared to controls (Supplemental Fig. 6C, D).

\section{Antibiotic treatment leads to accelerated tumor growth and attenuated sensitivity to cisplatin in patient derived EOC.}

To ensure our observed phenotype was not unique to syngeneic EOC cell lines (ID8 and ID8-VEGF). We repeated our study paradigm in NSG mice with a human derived OV81 cell line. Following 2 weeks of $A B X$ or control water, mice were IP injected with $5 \times 10^{6}$ OV81 cells. To monitor tumor growth is this cohort of mice, cells were transduced with a luciferase reporter prior to IP injection. Following 2 weeks IP cell injection, mice were randomized into 4 groups: $\mathrm{H}_{2} \mathrm{O}$ Vehicle, $\mathrm{H}_{2} \mathrm{O}$ Cisplatin, $\mathrm{ABX}$ Vehicle, and $\mathrm{ABX}$ Cisplatin and treated as previously outlined. At endpoint, mice underwent Perkin Elmer in Vivo Imaging System (IVIS) imaging and total flux of photons/second was calculated to determine total tumor burden. Overall $A B X$ therapy resulted in a drastic increase in total tumor burden when normalized to initial tumor burden compared to $\mathrm{H}_{2} \mathrm{O}$ controls in both the vehicle and cisplatin treated groups (Supplemental Fig. 7).

\section{ABX therapy induces a stem cell phenotype in ID8 EOC tumors in NSG mice}

To better understand the effect of antibiotic therapy on tumor cells and their growth in the presence and absence of cisplatin, we performed RNAseq on tumors collected from $\mathrm{H}_{2} \mathrm{O}$ Placebo, $\mathrm{H}_{2} \mathrm{O}$ Cisplatin, ABX Placebo, and ABX Cisplatin treated NSG mice at endpoint (8 weeks post ID8 cell injection). The top 100 most significantly affected genes by $p$ value following a pairwise comparison between the $\mathrm{H}_{2} \mathrm{O}$ Placebo and ABX Placebo groups (Fig. 5A) were analyzed utilizing DAVID software for the enrichment of GO terms. Interestingly, the most enriched GO terms included cell differentiation, proliferation, and locomotor activity in line with the increased self-renewal ability observed in the previous tumor sphere initiation assays (Fig. 5B). Genes commonly associated with cancer stem cell signatures were also increased in the $A B X$ treatment group compared to the $\mathrm{H}_{2} \mathrm{O}$ control group such as SOX2, WNT7a, HOXb5, HOXb6, DLX5, MSX1, EFNA4, SALL1, and PAX2 genes. SHOX2 and GATA5 genes that promote cell differentiation were also significantly decreased (Fig. 5C) (39-46). Upon KEGG pathway analysis of genes with a p-value $<0.01$ in the $A B X$ Placebo group vs. the $\mathrm{H}_{2} \mathrm{O}$ Placebo group, multiple signaling pathways were implicated including PI3-Akt, MAPK, WNT, and Hedgehog (Fig. 5D) (47-49).

As we observed increased stem cell markers based on RNAseq of tumors, we performed tumorsphere assays to assess stem cell frequency in tumors from $\mathrm{H}_{2} \mathrm{O}$ and $\mathrm{ABX}$ treated mice without and with cisplatin. Following endpoint necropsy, 
tumor tissue from each group was dissociated to single cells and plated in a tumor sphere formation assay. Following 14 days of incubation, sphere initiation frequency was determined demonstrating a significant increase in sphere initiation frequency of ID8 cells from $A B X$ treated $\mathrm{BL} / 6$ mice compared to control $\mathrm{H}_{2} \mathrm{O}$ in both the presence and absence of cisplatin therapy (Fig. 6A). This observed effect was increasingly evident in the NSG cohorts demonstrating an apparent increase in the stem cell population following $A B X$ therapy, further increased in the presence of cisplatin (Fig. 6B).

\section{Discussion}

In recent years, there has been growing evidence demonstrating a link between the gut microbiome, carcinogenesis and response to cancer therapy $(11-18)$. Studies have emerged supporting, in both pre-clinical models and patient cohorts, that antibiotic therapy associated gut microbial disruption may negatively impact the efficacy of immune checkpoint inhibitors and systemic anti-cancer drugs, including platinum chemotherapy (12-17). To date, the impact of the gut microbiome upon response to chemotherapy in women with gynecologic malignancies is yet to be explored. In women diagnosed with EOC, platinum chemotherapy remains the standard treatment in primary and newly recurrent disease. Disease prognosis and treatment efficacy depends upon platinum sensitivity, with platinum resistance portending a poor prognosis with limited active treatment options. Interventions to increase platinum sensitivity and prevent development of resistance are essential to improving the care of women with EOC.

The pre-clinical studies described in this manuscript demonstrate that antibiotic therapy, and associated changes within the gut microbiome, resulted in accelerated tumor growth, attenuated cisplatin sensitivity and decreased survival. In mice who received ABX, 16S rDNA analysis demonstrated that the gut microbiome was markedly altered, compared to controls. Specifically, significantly reduced diversity in the fecal microbial communities was noted regardless of cisplatin therapy. These findings are consistent with prior studies investigating the impact of the gut microbiome upon response to platinum chemotherapy and immune checkpoint inhibitor treatment for non-gynecologic cancers. Indeed, Routy et al, showed resistance to immune checkpoint inhibitors was linked to abnormal gut microbiome composition (18). Following disruption of the gut microbiome through ABX treatment, response to CpGoligonucleotide immunotherapy and cyclophosphamide was impaired secondary to reduced cytokine production, lower production of reactive oxygen species and diminished cytotoxicity. In a separate study, lida et al., demonstrated that disruption of the gut microbiome through $A B X$, impaired platinum response, with decreased tumor regression and survival, in animal models of colon cancer and lymphoma (14).

Similar findings have been documented in cohorts of patients undergoing platinum chemotherapy for non-gynecologic cancers. Among 800 patients enrolled on clinical trials receiving cyclophosphamide or cisplatin for chronic lymphocytic leukemia or lymphoma, receipt of ABX targeting gram positive species during chemotherapy was associated with significantly decreased treatment response, time to recurrence and survival (17). These findings suggest that certain presence of specific bacterial populations may be essential for treatment response. In our studies, we identified significantly higher relative abundance of potentially pathogenic Gram-negative Enterobacteriaceae and Parasutterella species. Notably, among animals treated with cisplatin, these bacteria were further increased, in the absence of facultative Gram-positive bacteria, to those who received placebo. Although not yet studied in relation to cancer, an increase in the abundance of Parasutterella has been associated with dysbiosis of the gut microbiome and alterations in liver metabolism $(37,38,50)$.

Furthermore, in NSG mice, we identified that disruption of the immune system does not directly contribute to increased tumor growth or reduced platinum sensitivity in the setting of microbial disruption. In addition, no differences in myeloid and lymphoid populations were observed in the ascitic fluid of mice following antibiotic treatment, compared 
to controls. Our results identify that in the setting of an altered microbiome, response to platinum chemotherapy is reduced and populations of chemo-resistance cancer stem cells are increased through tumorsphere initiation frequency and transcriptional reprogramming to an increased cancer stem cell-like state. The relationship between enhanced populations of cancer stem cells and platinum resistance in EOC is well-documented in the literature $(28,45,48,51-$ 54). Specifically, we identified SOX2, WNT7a, HOXb5, HOXb6, DLX5, MSX1, EFNA4, SALL 1, and PAX2 as being significantly upregulated in ID8 tumors of the $A B X$ group over the control $\mathrm{H}_{2} \mathrm{O}$ group. Many of these genes are markers

of pluripotency and regulation as well as markers of long-term stemness $(39,40,42-45)$. SALL1 interacts with NANOG a well-established pluripotency transcription factor, to suppress differentiation (44). Conversely, SHOX2 and GATA5 genes that favor differentiation, were significantly decreased providing further evidence that ID8 tumor cells from $A B X$ mice are more stem like and undifferentiated than ID8 tumor cells from $\mathrm{H}_{2} \mathrm{O}$ treated mice $(41,46,55)$. Upon KEGG pathway analysis of enriched genes in the ABX group, multiple signaling pathways such as P13K/AKT/mTOR and WNT, were implicated and currently under investigation as targets for ovarian cancer therapies $(49,56)$. Further investigation is needed to understand the mechanism through which the gut microbiome interacts with cancer stem cells and how this specifically drives platinum resistance in EOC.

The clinical implications of the gut microbiome impacting platinum response in EOC are significant. Primarily, antibiotic therapy is often unavoidable in the care of patients with EOC following cytoreductive surgery or during chemotherapy. However, evidence supporting that antibiotic driven changes to the gut microbiome may negatively impact oncologic outcomes supports that physicians should be judicious in antibiotic selection and duration of dosing and escalate treatment where appropriate. It also supports that measures to prevent infection in women with EOC, and therefore avoid antibiotic treatments, should be prioritized. Most importantly, understanding that disruption of gut microbiota impacts EOC growth and platinum chemotherapy introduces the potential for development of targeted therapeutics, designed to restore an intact gut microbiome, which may represent promising strategies to treat EOC and combat platinum resistance in the future.

Collectively, we identify a role for the disruption of gut microbiota in enhanced tumor growth and reduced sensitivity to platinum chemotherapy in pre-clinical models of EOC. Further investigation is critically needed to understand how the tumor microenvironment, and cancer stem cells specifically, communicate with the gut microbiome to drive platinum resistance in EOC. Answers to these questions will provide important insights as to whether microbe directed interventions to the gut microbiome can be used to impact the response to platinum chemotherapy.

\section{Conclusion}

Epithelial ovarian cancers are diagnosed at an advanced stage and patients exhibit poor overall survival of around two years. In preparation of patients for de-bulking surgery and during management of their disease, broad spectrum antibiotics are used as preventive and prophylactic treatment. It is well appreciated that the microbiome impacts human health and disease and the use of broad spectrum antibiotics leads to dysbiosis or a microbial imbalance. Our findings using mouse models of ovarian cancer provide evidence that antibiotic treatment leads to accelerated tumor growth and resistance to platinum based chemotherapy independent of immune status. Moreover, antibiotic treatment led to increased frequency of tumor initiating cells, known to exhibit poor response to chemotherapy. RNAseq analysis complements these findings showing an increase in expression of tumor initiating/cancer stem cell genes. These findings are directly translatable to patient care and the need to evaluate the broad use of antibiotics in care for epithelial ovarian cancer patients.

\section{Methods}


ID8, ID8-VEGF (syngeneic) and OV81 (human) EOC cell lines were cultured in Dulbecco Modified Eagle Medium (DMEM) media containing heat inactivated 5\% FBS (Atlas Biologicals Cat \# F-0500-D, Lot F31E18D1) and grown under standard conditions. HEK 293T/17 (ATCC CRL-11268) cells were plated and co-transfected with Lipofectamine 3000 (L3000015 Invitrogen), 3rd generation packaging vectors pRSV-REV \#12253, pMDG.2 \#12259, and pMDLg/pRRE \#12251 (Addgene) and lentiviral vector directing expression of luciferase reporter pHIV-Luciferase \#21375 $4.5 \mu \mathrm{g}$ (Addgene). Viral particles were harvested, filtered through a $0.45 \mu \mathrm{m}$ Durapore PVDF Membrane (Millipore SE1M003M00) and added to cell line culture media. Viral infections were carried out over 72 hours and transduced cells were selected by their resistance to $2 \mu \mathrm{g} / \mathrm{mL}$ puromycin (MP Biomedicals 0219453910).

\section{$\underline{\text { Animal Studies }}$}

Female C57BL/6J (BL/6) mice were purchased from Jackson Laboratories (Bar Harbor, ME) at 6 weeks of age. Female NOD.Cg-Prkdc<scid $>\| 2$ rg $<$ tm1 Wjl>SzJ (NSG) mice were purchased from the Cleveland Clinic BRU and Gnotobiotic Core Facility respectively at 6 weeks of age. Experimental animals were housed and handled in accordance with Cleveland Clinic Lerner Research Institute IACUC guidelines. Mice (BL/6 or NSG) were given either control or antibiotic ( $0.5 \mathrm{~g} / \mathrm{L}$ vancomycin, $1 \mathrm{~g} / \mathrm{L}$ neomycin sulfate, $1 \mathrm{~g} / \mathrm{L}$ metronidazole, $1 \mathrm{~g} / \mathrm{L}$ ampicillin) (ABX) (Fisher Scientific) containing water for 2 weeks prior to intraperitoneal (IP) injection of ID8-LUC $\left(5 \times 10^{6}\right)$ ID8-VEGF $\left(5 \times 10^{6}\right)$ or OV81 $\left(1 \times 10^{6}\right)$ cells. The ABX containing water has been previously shown to be sufficient to deplete all detectable commensal bacteria $(32,33)$. Mice remained on either control or ABX containing water for the duration of the study. At 10 weeks of age, mice were treated IP with either cisplatin (5mg/kg, Spectrum Chemical or vehicle (PBS) weekly until human endpoint of total tumor burden exceeding $150 \mathrm{~mm}^{3}$ or debilitating ascites development.

\section{Tumor Monitoring $\underline{\text { by }}$ Trans abdominal Ultrasound (TAUS).}

For the ID8-LUC and ID8-VEGF cohorts, TAUS surveillance was initiated seven days following IP tumor injection as previously described (22). TAUS was performed every 10 days until study endpoint. Mice were anesthetized using isoflurane (DRE Veterinary) and placed in the supine position. Following the removal of abdominal hair using Nair (Church \& Dwight Co. Inc.), sterile ultrasound gel was applied to the abdomen. TAUS was performed using Vevo2100 (VisualSonics) using the abdominal imaging package and MS550D probe $(40 \mathrm{~Hz})$. For each mouse, the abdomen was assessed for tumor in four quadrants. Tumors were noted to be absent or present at each assessment. Tumor length and width were recorded and tumor volume was calculated using the formula: (Length^(Width^2))/2.

\section{Tumor Monitoring by 2 2D IVIS Imaging.}

For the A2780-LUC cohort of mice, bioluminescence images were taken with IVIS Lumina (PerkinElmer) using Dluciferin as previously described (57). Mice received an IP injection of D-luciferin (Goldbio LUCK-1G, 150mg/kg in 150uL) under inhaled isoflurane anesthesia. Images were analyzed (Living Image Software) and total flux reported in photons/second/ $\mathrm{cm}^{\wedge} 2 /$ steradian for each mouse abdomen. All images were obtained with a 15 second exposure.

\section{S rDNA Sequencing}

At 5 pre-determined time points (baseline, 2 weeks, tumor engraftment, 5 weeks, and endpoint, see Supplemental Figure 1 schematic), stool was collected from each mouse and frozen in micro centrifuge tubes at -80C. Upon completion of the study, 16S rDNA was isolated following the standard protocol and (QIAamp PowerFecalPro Kit) (Qiagen). Isolated samples were sent to Miami University of Ohio on dry ice for 16S V4 rDNA processing. Data analysis was completed by the Microbiome and Composition Analytics Core facility as follows: Individual fastq files without non-biological 
nucleotides were processed using Divisive Amplicon Denoising Algorithm (DADA) pipeline (58). The output of the dada2 pipeline (feature table of amplicon sequence variants (an ASV table)) was processed for alpha and beta diversity analysis using phyloseq (59), and microbiomeSeq (http://www.github.com/umerijaz/microbiomeSeq) packages in R. Alpha diversity estimates were measured within group categories using estimate_richness function of the phyloseq package (59). Multidimensional scaling (MDS, also known as principal coordinate analysis; PCoA) was performed using Bray-Curtis dissimilarity matrix (60) between groups and visualized by using ggplot2 package (61). We assessed the statistical significance $(P<0.05)$ throughout and whenever necessary, we adjusted $P$-values for multiple comparisons according to the Benjamini and Hochberg method to control False Discovery Rate (62) while performing multiple testing on taxa abundance according to sample categories. We performed an analysis of variance (ANOVA) among sample categories while measuring the of a-diversity measures using plot_anova_diversity function in microbiomeSeq package (http://www.github.com/umerijaz/microbiomeSeq). Permutational multivariate analysis of variance (PERMANOVA) with 999 permutations was performed on all principal coordinates obtained during PCoA with the ordination function of the microbiomeSeq package. Linear regression (parametric test), and Wilcoxon (Nonparametric) test were performed on ASVs abundances against coprostanol levels using their base functions in R (63).

\section{Tumor RNA Sequencing.}

Tumors were collected at endpoint from NSG ID8 mice and snap frozen in liquid nitrogen. Prior to RNA extraction (Takara Nucleospin), tumors were crushed using ice-cold mortar and pestle under sterile conditions. Following RNA isolation, cDNA libraries were prepared by the LRI Genomics Core facility and sent to Macrogen Inc. for RNA sequencing. The RNA sequencing results were analyzed by the Cleveland Clinic Quantitative Health Sciences' Bioinformatics Consulting Service as follows:

Raw sequence FASTQ files underwent read quality assessment with FASTQC (version 0.11.8, https://www.bioinformatics.babraham.ac.uk/projects/fastqc/) (64). Ribosomal RNA content was

evaluated with SortMeRNA (version 2.1) (65). Adapter removal and quality trimming was performed with Trimgalore (version 0.5.0, https://github.com/FelixKrueger/TrimGalore). Adapter removed, trimmed reads were aligned to the Mus musculus genome assembly (GRCm38 version 99,

ftp://ftp.ensembl.org/pub/release99/fasta/mus_musculus/dna/Mus_musculus.GRCm38.dna.primary_assembly.fa.gz) using STAR (version 2.6.1d)(66), and duplicates were marked with picard (version 2.18.27, http://broadinstitute.github.io/picard/). Alignments were assessed for quality with qualimap (version 2.2.2)(67) and rseqc (version 3.0.0)(68), and for library complexity saturation with preseq (version 2.0.3)(69). Aligned reads were counted at the exon level and summarized at the gene level with the featureCounts tool from the Subread package (version 1.6.4)(70), using annotations for build GRCm38 (version99,ftp://ftp.ensembl.org/pub/release99/gtf/mus_musculus/Mus_musculus.GRCm38.99.gtf.gz). Normalization and differential expression analysis was performed with the R (version 3.6.3)(71) package DESeq2 (version 1.26.0) (72). Size factor and dispersion estimation were performed with default settings. Comparison estimate p-values for $\mathrm{H}_{2} \mathrm{O}$-Cisplatin vs. $\mathrm{H}_{2} \mathrm{O}$-Placebo, Antibiotic-Cisplatin vs. Antibiotic-Placebo, Antibiotic-Cisplatin vs. $\mathrm{H}_{2} \mathrm{O}-\mathrm{Cisplatin}$, and Antibiotic-Placebo vs. $\mathrm{H}_{2} \mathrm{O}$-Placebo were extracted using multiple testing adjustment with the method of Benjamini and Hochberg (73), and an independent filtering significance cut-off of 0.05 (74). Log2-fold change estimates for each comparison were shrunken with the function IfcShrink from the DESeq2 package, using default settings. The web tool DAVID bioinformatics database was utilized to assess the top 100 significantly expressed genes from each group comparison for enrichment of GO terms (75).

\section{Flow Cytometry}


Upon murine necropsy, ascites was collected and centrifuged cells were stored in freezing media (10\% DMSO in FBS, Atlas Biologicals) at $-80 \mathrm{C}$. All single cells suspensions were thawed on ice, washed once with $2 \% \mathrm{BSA}$ in PBS and stained with live/dead UV stain (Invitrogen) and then blocked in FACS buffer (PBS, 2\% BSA) containing FcR blocking reagent at 1:50 (Miltenyi Biotec) for 15 minutes. After live/dead staining and blocking, antibody cocktails for myeloid or lymphoid panels (Supplemental Figure 2 A), were incubated with cells at a 1:50 dilution for 20 minutes on ice before being washed and suspended in FACS buffer for analysis.

Cell populations were analyzed using an LSRFortessa (BD Biosciences), and populations were separated and quantified using FlowJo software (Tree Star Inc.). Gating methods for myeloid and lymphoid populations were performed following standardized gating strategies previously described $(76,77)$. For a complete gating strategies please see Supplemental Figure 2 B.

\section{Immunohistochemical $(\underline{\mathrm{IHC}})$ Staining}

At endpoint, tumors were excised and placed in 4\% paraformaldehyde (Electron Microscopy Sciences), at room temperature for 24-48 hours and subsequently transferred to $70 \%$ ethanol and stored at $4 \mathrm{C}$. Samples were then placed in paraffin blocks, sectioned, and stained with hematoxylin and eosin by the Cleveland Clinic Foundation Histology Core to confirm EOC phenotype.

\section{Tumorsphere Formation Studies}

Upon murine necropsy, Total tumor collected per mouse was dissociated using standard methods with a Papain dissociation kit (Worthington Biologicals). Following filtration through a 40 micron filter, single cells were counted cultured in serial dilutions in a non-adherent 96-well plate (Sarstedt) with $200 \mu$ l serum-free DMEM/F12 medium supplemented with $20 \mathrm{ng} / \mathrm{mL}$ basic fibroblast growth factor (Invitrogen), $10 \mathrm{ng} / \mathrm{mL}$ epidermal growth factor (BioSource), and 2\% B27 (vol/vol) (Invitrogen). Tumorsphere-formation was scored following 2 weeks incubation using a phase contrast microscope. The sphere initiation frequency was calculated using an extreme limiting dilution algorithm (ELDA) (http://bioinf.wehi.edu.au/software/elda/).

\section{$\underline{\text { In vitro } A B X \text { cisplatin sensitivity studies }}$}

ID8-LUC and ID8-VEGF cells were plated at 1,000 cells per well and treated with ampicillin, vancomycin, metrinodazole, and neomycin in combinations of the same ratios used in the murine studies for 72 hours, wherein proliferation was measured using Cell Titre Glo (Promega) and compared to vehicle treated control. Additionally following incubation for 7 days with ampicillin, vancomycin, metrinodazole, and neomycin the IC50 of cisplatin (Spectrum Chemical) was assessed compared to vehicle pretreated controls also utilizing Cell Titre Glo (Promega).

\section{Statistics}

All data are presented as mean +/- standard error of the mean. All statistical analysis was performed in GraphPad Prism v8. Replicate numbers and p-values are presented in figure legends.

\section{Study Approvals}

All murine studies were completed in accordance with the Institutional Animal Care and Use Committee guidelines, approval \# 2018-2003. All studies utilizing lenti-viral particle generation were completed in accordance with the Institutional Biosafety Committee guidelines, approval \#IBC0920.

\section{Declarations}


Consent for publication-Not Applicable

Availability of data and material- Metagenomic and transcriptomic data generated and analyzed pertaining to the presented studies will be made publicly available in the NCBI GEO database.

Competing Interests- The authors declare that they have no competing interests.

Funding- Not Applicable

Author's Contributions- LC: concept and study design, animal experiments, data analysis and interpretation, writing/editing of manuscript, EE: study design, animal experiments, data analysis and interpretation, writing/editing of manuscript, CB: animal experiments, data collection, and editing of manuscript, LT: animal experiments and data collection, ZA: animal experiments, data collection and analysis, editing of manuscript, DB: flow cytometry data analysis, experimental design, and writing/editing manuscript, JL: writing/editing manuscript and technical/experimental insights, NS: analysis of $16 \mathrm{~S}$ sequencing, writing/editing manuscript, PB: RNAseq data analysis and writing/editing manuscript, AJP: histology specimen analysis, MD: writing/editing manuscript and technical/experimental insights, $\mathrm{AH}$ : writing/editing manuscript and technical/experimental insights, PA: writing/editing manuscript and technical/experimental insights, JC: writing/editing manuscript and technical/experimental insights, PR: writing/editing manuscript, RV: writing of manuscript, conceptualization of studies, CM: conceptualization of studies and writing/editing manuscript, OR: conceptualization of studies, experimental design and analysis oversight, resource allocation for performed experiments, writing/editing of manuscript.

\section{Acknowledgements}

The authors would like to acknowledge members of the Reizes laboratory for their collaborative effort and insight toward the completion of the studies within this manuscript, as well as the LRI Core Facilities that played a role in data collection, analysis, or interpretation of the findings presented within the manuscript including: Microbiome Analytics and Composition Core Facility, Imaging Core, Histology Core, and Microbial Culture and Engineering Core. DB received support from NIH F32 CA243314. Dr. Reizes is the Laura J. Fogarty Endowed Chair in Uterine Cancer Research. Research in the Reizes laboratory is funded by VeloSano Bike to Cure, Center of Research Excellence in Gynecologic Cancer, and the Department of Defense.

\section{References}

1. Siegel R, Naishadham D, Jemal A. Cancer statistics, 2012. CA Cancer J Clin. 2012;62(1):10-29.

2. Bian C, Yao K, Li L, Yi T, Zhao X. Primary debulking surgery vs. neoadjuvant chemotherapy followed by interval debulking surgery for patients with advanced ovarian cancer. Arch Gynecol Obstet. 2016;293(1):163-8.

3. Heintz AP, Odicino F, Maisonneuve P, Quinn MA, Benedet JL, Creasman WT, et al. Carcinoma of the ovary. FIGO 26th Annual Report on the Results of Treatment in Gynecological Cancer. Int J Gynaecol Obstet. 2006;95 Suppl 1:S161-92.

4. Kehoe S, Hook J, Nankivell M, Jayson GC, Kitchener H, Lopes T, et al. Primary chemotherapy versus primary surgery for newly diagnosed advanced ovarian cancer (CHORUS): an open-label, randomised, controlled, noninferiority trial. Lancet. 2015;386(9990):249-57. 
5. Morrison J, Haldar K, Kehoe S, Lawrie TA. Chemotherapy versus surgery for initial treatment in advanced ovarian epithelial cancer. Cochrane Database Syst Rev. 2012(8):CD005343.

6. Pignata S, Scambia G, Katsaros D, Gallo C, Pujade-Lauraine E, De Placido S, et al. Carboplatin plus paclitaxel once a week versus every 3 weeks in patients with advanced ovarian cancer (MITO-7): a randomised, multicentre, openlabel, phase 3 trial. Lancet Oncol. 2014;15(4):396-405.

7. Siesto G, Cavina R, Romano F, Vitobello D. Primary Debulking Surgery Versus Neoadjuvant Chemotherapy in Advanced Epithelial Ovarian Cancer: A Propensity-matched Analysis. Am J Clin Oncol. 2018;41(3):280-5.

8. Vergote I, Trope CG, Amant F, Kristensen GB, Ehlen T, Johnson N, et al. Neoadjuvant chemotherapy or primary surgery in stage IIIC or IV ovarian cancer. N Engl J Med. 2010;363(10):943-53.

9. Hanker LC, Loibl S, Burchardi N, Pfisterer J, Meier W, Pujade-Lauraine E, et al. The impact of second to sixth line therapy on survival of relapsed ovarian cancer after primary taxane/platinum-based therapy. Ann Oncol. 2012;23(10):2605-12.

10. Bhatt AP, Redinbo MR, Bultman SJ. The role of the microbiome in cancer development and therapy. CA Cancer J Clin. 2017;67(4):326-44.

11. Gopalakrishnan V, Helmink BA, Spencer CN, Reuben A, Wargo JA. The Influence of the Gut Microbiome on Cancer, Immunity, and Cancer Immunotherapy. Cancer Cell. 2018;33(4):570-80.

12. Gopalakrishnan V, Spencer CN, Nezi L, Reuben A, Andrews MC, Karpinets TV, et al. Gut microbiome modulates response to anti-PD-1 immunotherapy in melanoma patients. Science. 2018;359(6371):97-103.

13. Goubet AG, Daillere R, Routy B, Derosa L, Zitvogel PMR. L. The impact of the intestinal microbiota in therapeutic responses against cancer. C R Biol. 2018;341(5):284-9.

14. lida N, Dzutsev A, Stewart CA, Smith L, Bouladoux N, Weingarten RA, et al. Commensal bacteria control cancer response to therapy by modulating the tumor microenvironment. Science. 2013;342(6161):967-70.

15. Kuczma MP, Ding ZC, Li T, Habtetsion T, Chen T, Hao Z, et al. The impact of antibiotic usage on the efficacy of chemoimmunotherapy is contingent on the source of tumor-reactive T cells. Oncotarget. 2017;8(67):111931-42.

16. Matson V, Fessler J, Bao R, Chongsuwat T, Zha Y, Alegre ML, et al. The commensal microbiome is associated with anti-PD-1 efficacy in metastatic melanoma patients. Science. 2018;359(6371):104-8.

17. Pflug N, Kluth S, Vehreschild JJ, Bahlo J, Tacke D, Biehl L, et al. Efficacy of antineoplastic treatment is associated with the use of antibiotics that modulate intestinal microbiota. Oncoimmunology. 2016;5(6):e1150399.

18. Routy B, Le Chatelier E, Derosa L, Duong CPM, Alou MT, Daillere R, et al. Gut microbiome influences efficacy of PD1-based immunotherapy against epithelial tumors. Science. 2018;359(6371):91-7.

19. Heshiki Y, Vazquez-Uribe R, Li J, Ni Y, Quainoo S, Imamovic L, et al. Predictable modulation of cancer treatment outcomes by the gut microbiota. Microbiome. 2020;8(1):28.

20. Tran CW, McGree ME, Weaver AL, Martin JR, Lemens MA, Cliby WA, et al. Surgical site infection after primary surgery for epithelial ovarian cancer: predictors and impact on survival. Gynecol Oncol. 2015;136(2):278-84.

21. Chambers LMKM, Esakov E, Braley C, Sangwan N, Michener CM, Rose PG, Debernardo R, Vargas R, Reizes 0. Antibiotic Therapy Worsens Outcomes Following Primary Treatment in Ovarian Cancer: Potential Role of the Gut Microbiome. 25. Colorado: Annual Winter Meeting of the Society of Gynecologic Oncology; Aspen; 2020. th .

22. Chambers LM, Esakov E, Braley C, AlHilli M, Michener C, Reizes O. Use of Transabdominal Ultrasound for the detection of intra-peritoneal tumor engraftment and growth in mouse xenografts of epithelial ovarian cancer. PLoS One. 2020;15(4):e0228511.

23. Holtz DO, Krafty RT, Mohamed-Hadley A, Zhang L, Alagkiozidis I, Leiby B, et al. Should tumor VEGF expression influence decisions on combining low-dose chemotherapy with antiangiogenic therapy? Preclinical modeling in 
ovarian cancer. J Transl Med. 2008;6:2.

24. Wilkinson-Ryan I, Pham MM, Sergent P, Tafe LJ, Berwin BL. A Syngeneic Mouse Model of Epithelial Ovarian Cancer Port Site Metastases. Transl Oncol. 2019;12(1):62-8.

25. Cohen M, Petignat P. The bright side of ascites in ovarian cancer. Cell Cycle. 2014;13(15):2319.

26. Kipps E, Tan DS, Kaye SB. Meeting the challenge of ascites in ovarian cancer: new avenues for therapy and research. Nat Rev Cancer. 2013;13(4):273-82.

27. Cheng H, Wang Z, Cui L, Wen Y, Chen X, Gong F, et al. Opportunities and Challenges of the Human Microbiome in Ovarian Cancer. Front Oncol. 2020;10:163.

28. Kovacs T, Miko E, Ujlaki G, Sari Z, Bai P. The Microbiome as a Component of the Tumor Microenvironment. Adv Exp Med Biol. 2020;1225:137-53.

29. Zhou B, Sun C, Huang J, Xia M, Guo E, Li N, et al. The biodiversity Composition of Microbiome in Ovarian Carcinoma Patients. Sci Rep. 2019;9(1):1691.

30. Drakes ML, Stiff PJ. Regulation of Ovarian Cancer Prognosis by Immune Cells in the Tumor Microenvironment. Cancers (Basel). 2018;10(9).

31. Ishikawa F, Yasukawa M, Lyons B, Yoshida S, Miyamoto T, Yoshimoto G, et al. Development of functional human blood and immune systems in NOD/SCID/IL2 receptor \{gamma\} chain(null) mice. Blood. 2005;106(5):1565-73.

32. Rakoff-Nahoum S, Paglino J, Eslami-Varzaneh F, Edberg S, Medzhitov R. Recognition of commensal microflora by toll-like receptors is required for intestinal homeostasis. Cell. 2004;118(2):229-41.

33. Wang Z, Klipfell E, Bennett BJ, Koeth R, Levison BS, Dugar B, et al. Gut flora metabolism of phosphatidylcholine promotes cardiovascular disease. Nature. 2011;472(7341):57-63.

34. Kim S, Covington A, Pamer EG. The intestinal microbiota: Antibiotics, colonization resistance, and enteric pathogens. Immunol Rev. 2017;279(1):90-105.

35. Iredell J, Brown J, Tagg K. Antibiotic resistance in Enterobacteriaceae: mechanisms and clinical implications. BMJ. 2016;352:h6420.

36. Kirby A, Santoni N. Antibiotic resistance in Enterobacteriaceae: what impact on the efficacy of antibiotic prophylaxis in colorectal surgery? J Hosp Infect. 2015;89(4):259-63.

37. Chiodini RJ, Dowd SE, Chamberlin WM, Galandiuk S, Davis B, Glassing A. Microbial Population Differentials between Mucosal and Submucosal Intestinal Tissues in Advanced Crohn's Disease of the lleum. PLoS One. 2015;10(7):e0134382.

38. Huang C, Chen J, Wang J, Zhou H, Lu Y, Lou L, et al. Dysbiosis of Intestinal Microbiota and Decreased Antimicrobial Peptide Level in Paneth Cells during Hypertriglyceridemia-Related Acute Necrotizing Pancreatitis in Rats. Front Microbiol. 2017;8:776.

39. Chen JY, Miyanishi M, Wang SK, Yamazaki S, Sinha R, Kao KS, et al. Hoxb5 marks long-term haematopoietic stem cells and reveals a homogenous perivascular niche. Nature. 2016;530(7589):223-7.

40. Fischbach NA, Rozenfeld S, Shen W, Fong S, Chrobak D, Ginzinger D, et al. HOXB6 overexpression in murine bone marrow immortalizes a myelomonocytic precursor in vitro and causes hematopoietic stem cell expansion and acute myeloid leukemia in vivo. Blood. 2005;105(4):1456-66.

41. Ionta V, Liang W, Kim EH, Rafie R, Giacomello A, Marban E, et al. SHOX2 overexpression favors differentiation of embryonic stem cells into cardiac pacemaker cells, improving biological pacing ability. Stem Cell Reports. 2015;4(1):129-42.

42. Le Grand F, Jones AE, Seale V, Scime A, Rudnicki MA. Wnt7a activates the planar cell polarity pathway to drive the symmetric expansion of satellite stem cells. Cell Stem Cell. 2009;4(6):535-47. 
43. Taghiyar L, Hesaraki M, Sayahpour FA, Satarian L, Hosseini S, Aghdami N, et al. Msh homeobox 1 (Msx1)- and Msx2-overexpressing bone marrow-derived mesenchymal stem cells resemble blastema cells and enhance regeneration in mice. J Biol Chem. 2017;292(25):10520-33.

44. Karantzali E, Lekakis V, Ioannou M, Hadjimichael C, Papamatheakis J, Kretsovali A. Sall1 regulates embryonic stem cell differentiation in association with nanog. J Biol Chem. 2011;286(2):1037-45.

45. Nagare RP, Sneha S, Sidhanth C, Roopa S, Murhekar K, Shirley S, et al. Expression of cancer stem cell markers CD24, EPHA1 and CD9 and their correlation with clinical outcome in epithelial ovarian tumours. Cancer Biomark. 2020.

46. Xia L, Gong Y, Zhang A, Cai S, Zeng Q. Loss of GATA5 expression due to gene promoter methylation induces growth and colony formation of hepatocellular carcinoma cells. Oncol Lett. 2016;11(1):861-9.

47. Yang L, Shi P, Zhao G, Xu J, Peng W, Zhang J, et al. Targeting cancer stem cell pathways for cancer therapy. Signal Transduct Target Ther. 2020;5:8.

48. Sneha S, Nagare RP, Sidhanth C, Krishnapriya S, Garg M, Ramachandran B, et al. The hedgehog pathway regulates cancer stem cells in serous adenocarcinoma of the ovary. Cell Oncol (Dordr). 2020.

49. Gasparri ML, Bardhi E, Ruscito I, Papadia A, Farooqi AA, Marchetti C, et al. PI3K/AKT/mTOR Pathway in Ovarian Cancer Treatment: Are We on the Right Track? Geburtshilfe Frauenheilkd. 2017;77(10):1095-103.

50. Ju T, Kong JY, Stothard P, Willing BP. Defining the role of Parasutterella, a previously uncharacterized member of the core gut microbiota. ISME J. 2019;13(6):1520-34.

51. Sabini C, Sorbi F, Cunnea P, Fotopoulou C. Ovarian cancer stem cells: ready for prime time? Arch Gynecol Obstet. 2020;301(4):895-9.

52. Saygin C, Matei D, Majeti R, Reizes O, Lathia JD. Targeting Cancer Stemness in the Clinic: From Hype to Hope. Cell Stem Cell. 2019;24(1):25-40.

53. Saygin C, Wiechert A, Rao VS, Alluri R, Connor E, Thiagarajan PS, et al. CD55 regulates self-renewal and cisplatin resistance in endometrioid tumors. J Exp Med. 2017;214(9):2715-32.

54. Terraneo N, Jacob F, Dubrovska A, Grunberg J. Novel Therapeutic Strategies for Ovarian Cancer Stem Cells. Front Oncol. 2020;10:319.

55. Miyamoto T, Furusawa C, Kaneko K. Pluripotency, Differentiation, and Reprogramming: A Gene Expression Dynamics Model with Epigenetic Feedback Regulation. PLoS Comput Biol. 2015;11(8):e1004476.

56. Nguyen VHL, Hough R, Bernaudo S, Peng C. Wnt/beta-catenin signalling in ovarian cancer: Insights into its hyperactivation and function in tumorigenesis. J Ovarian Res. 2019;12(1):122.

57. Ryner L, Guan Y, Firestein R, Xiao Y, Choi Y, Rabe C, et al. Upregulation of Periostin and Reactive Stroma Is Associated with Primary Chemoresistance and Predicts Clinical Outcomes in Epithelial Ovarian Cancer. Clin Cancer Res. 2015;21(13):2941-51.

58. Callahan BJ, McMurdie PJ, Rosen MJ, Han AW, Johnson AJ, Holmes SP. DADA2: High-resolution sample inference from Illumina amplicon data. Nat Methods. 2016;13(7):581-3.

59. McMurdie PJ, Holmes S. phyloseq: an R package for reproducible interactive analysis and graphics of microbiome census data. PLoS One. 2013;8(4):e61217.

60. McMurdie PJ, Holmes S. Waste not, want not: why rarefying microbiome data is inadmissible. PLoS Comput Biol. 2014;10(4):e1003531.

61. Wickham H. ggplot2: Elegant Graphics for Data Analysis: Springer Publishing Company, Incorporated; 2009.216 p.

62. Benjamini Y. Discovering the false discovery rate. J Roy Stat Soc B. 2010;72(4):405-16. 
63. Tiit E-M. Nonparametric Statistical Methods. Myles Hollander and Douglas Wolfe A, Wiley, Chichester, 1999. No. of pages: xiii + 779. Price: $£$ 39.95. ISBN 0-471-19045-4. Statistics in Medicine. 2000;19(10):1386-8.

64. Bronte V, Brandau S, Chen SH, Colombo MP, Frey AB, Greten TF, et al. Recommendations for myeloid-derived suppressor cell nomenclature and characterization standards. Nat Commun. 2016;7:12150.

65. Kopylova E, Noe L, Touzet H. SortMeRNA: fast and accurate filtering of ribosomal RNAs in metatranscriptomic data. Bioinformatics. 2012;28(24):3211-7.

66. Dobin A, Davis CA, Schlesinger F, Drenkow J, Zaleski C, Jha S, et al. STAR: ultrafast universal RNA-seq aligner. Bioinformatics. 2013;29(1):15-21.

67. Okonechnikov K, Conesa A, Garcia-Alcalde F. Qualimap 2: advanced multi-sample quality control for highthroughput sequencing data. Bioinformatics. 2016;32(2):292-4.

68. Wang L, Wang S, Li W. RSeQC: quality control of RNA-seq experiments. Bioinformatics. 2012;28(16):2184-5.

69. Daley T, Smith AD. Predicting the molecular complexity of sequencing libraries. Nat Methods. 2013;10(4):325-7.

70. Liao Y, Smyth GK, Shi W. The Subread aligner: fast, accurate and scalable read mapping by seed-and-vote. Nucleic Acids Res. 2013;41(10):e108.

71. Team RDC. R: A language and environment for statistical computing. Vienna: R Foundation for Statistical Computing,; 2014.

72. Love MI, Huber W, Anders S. Moderated estimation of fold change and dispersion for RNA-seq data with DESeq2. Genome Biol. 2014;15(12):550.

73. Benjamini Y, Hochberg. Yosef. Controlling the false discovery rate: a practical and powerful approach to multiple testing. J Roy Stat Soc. 1995;57:289-300.

74. Bourgon R, Gentleman R, Huber W. Independent filtering increases detection power for high-throughput experiments. Proc Natl Acad Sci U S A. 2010;107(21):9546-51.

75. Huang da W, Sherman BT, Zheng X, Yang J, Imamichi T, Stephens R, et al. Extracting biological meaning from large gene lists with DAVID. Curr Protoc Bioinformatics. 2009;Chap. 13:Unit 131.

76. Alban TJ, Alvarado AG, Sorensen MD, Bayik D, Volovetz J, Serbinowski E, et al. Global immune fingerprinting in glioblastoma patient peripheral blood reveals immune-suppression signatures associated with prognosis. $\mathrm{JCl}$ Insight. 2018;3(21).

77. Bayik D, Zhou Y, Park C, Hong C, Vail D, Silver DJ, et al. Myeloid-derived suppressor cell subsets drive glioblastoma growth in a sex-specific manner. Cancer Discov. 2020.

\section{Figures}


A.

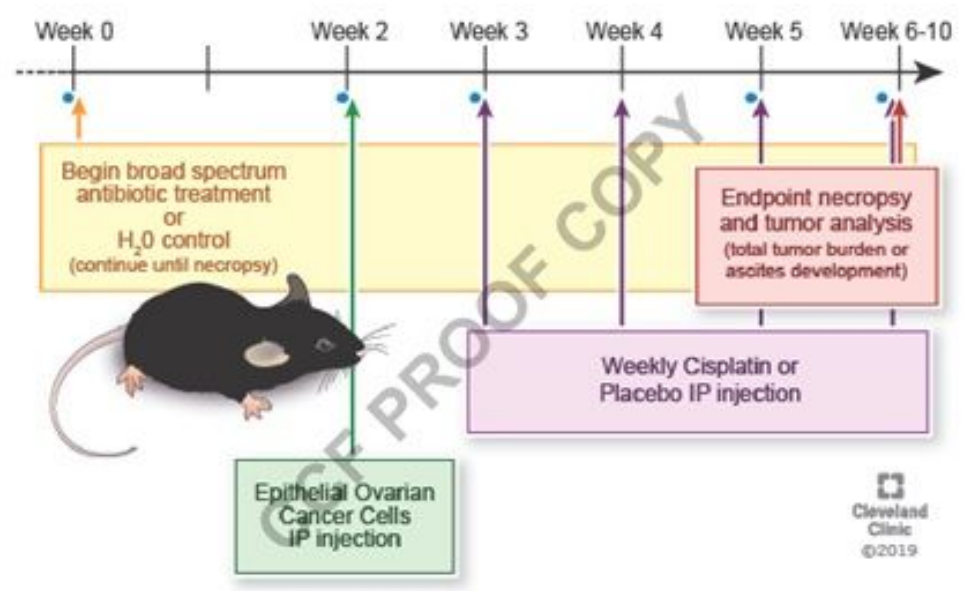

- Stool collection
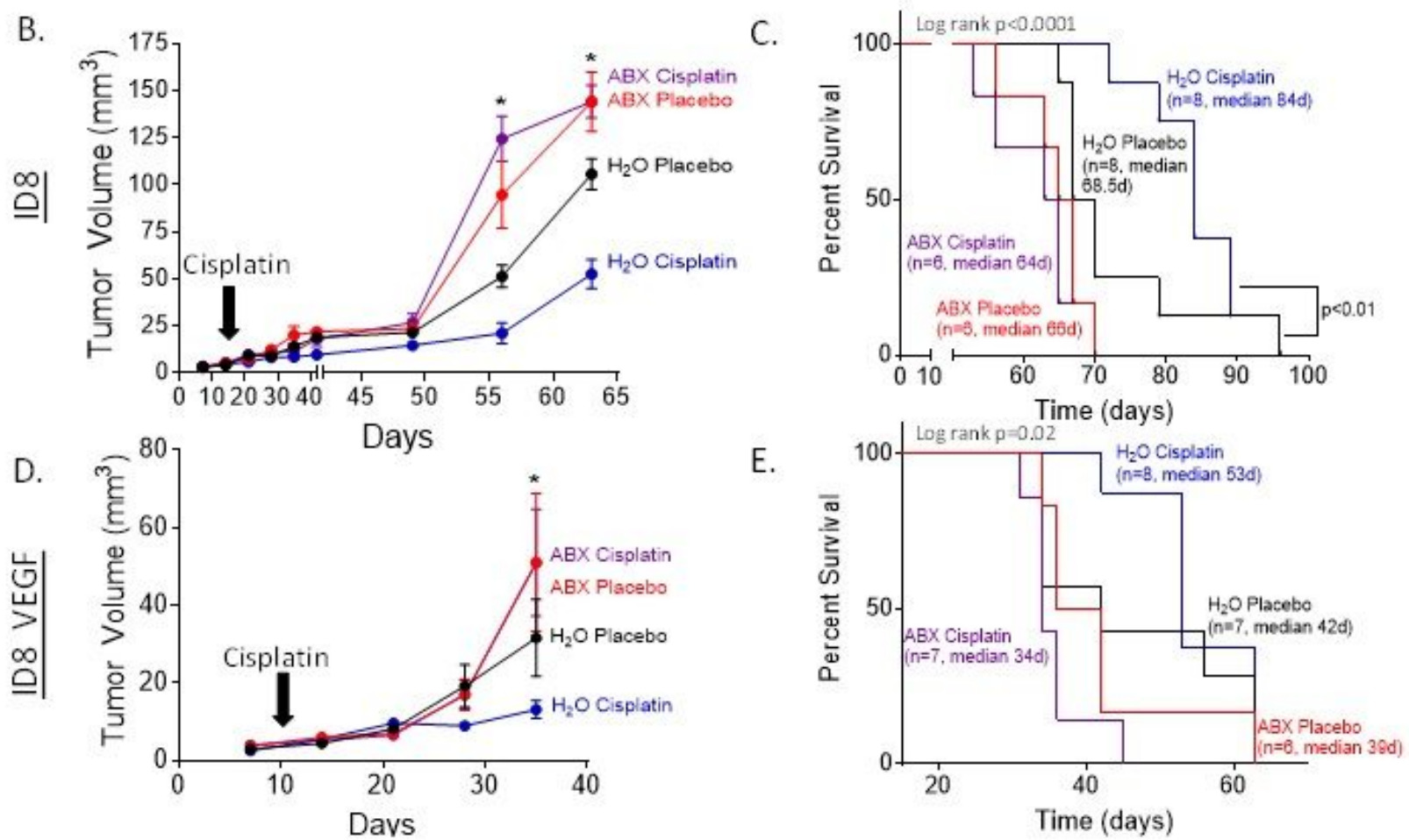

Figure 1

Tumor growth is increased and median survival is decreased in ABX treated $\mathrm{Bl} / 6$ mice, regardless of cisplatin therapy. Mice treated with antibiotics following the schematic study design $(A)$ exhibited increased tumor growth regardless of cisplatin therapy following injection of both ID8 (B) and ID8 VEGF (D) cells in C57 BL/6 mice. Antibiotic treated ID8 (C) and ID8 VEGF (E) injected C57 Bl/6 mice (purple and red) had significantly decreased median survival compared to $\mathrm{H} 2 \mathrm{O}$ treated mice (black and blue). (ABX Cis vs. H2O Cis, ID8 and ID8 VEGF p<0.0002) Further, cisplatin therapy increased $\mathrm{H} 2 \mathrm{O}$ treated murine survival (blue vs black, ID8 $\mathrm{p}<0.01$ ), but did not alter ABX treated murine survival (purple vs red), in either cohort. Statistical analysis: Log rank (Mantel Cox) test. $n=8$ mice per group 
A.
$\stackrel{\infty}{\emptyset}$

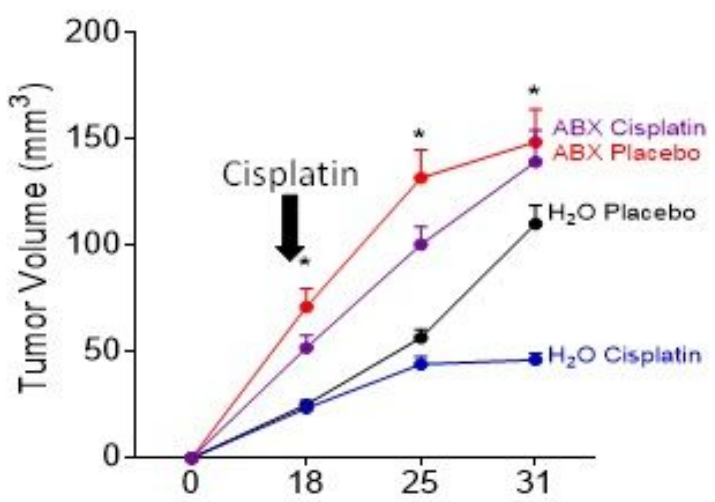

C.

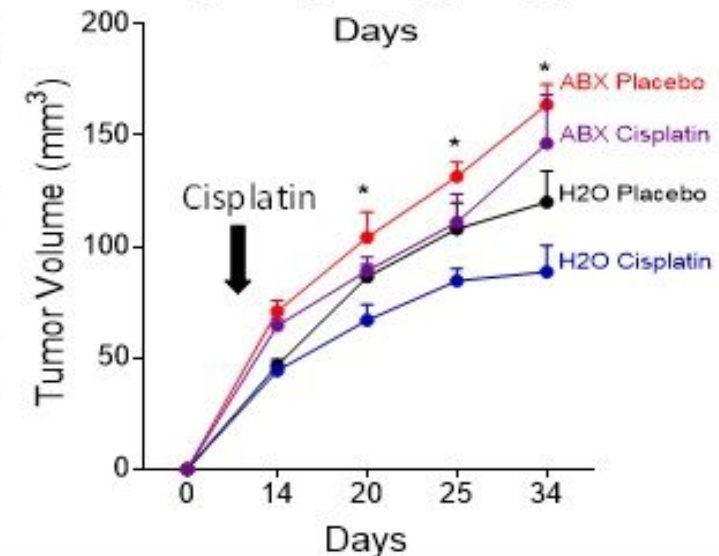

B.

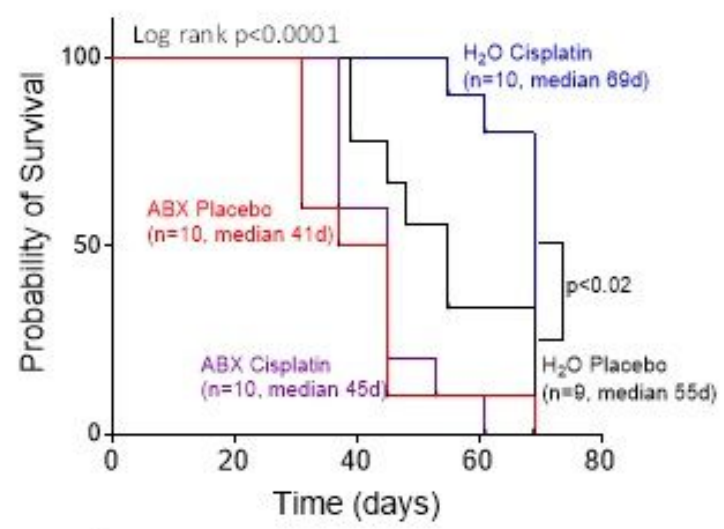

D.

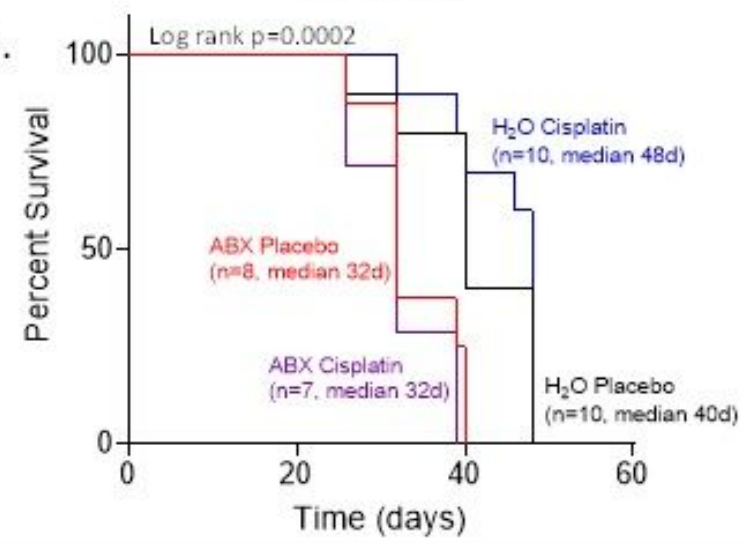

Figure 2

Tumor growth is increased and median survival is decreased in ABX treated mice, regardless of cisplatin therapy in immune-deficient NSG mice. Mice treated with antibiotics exhibited increased tumor growth regardless of cisplatin therapy following injection of both ID8 (A) and ID8 VEGF (C) cells in C57 BL/6 mice. Antibiotic treated ID8 (B) and ID8 VEGF (D) injected NSG mice (purple and red) had significantly decreased median survival compared to $\mathrm{H} 20$ treated mice (black and blue). Further, cisplatin therapy increased H2O treated murine survival (blue vs black), but did not alter $A B X$ treated murine survival (purple vs red) (ID8 $p<0.0001$, ID 8 VEGF $p<0.0005$ ), in either cohort. Statistical analysis: Log rank (Mantel Cox) test. $\mathrm{n}=8$ mice per group

A.

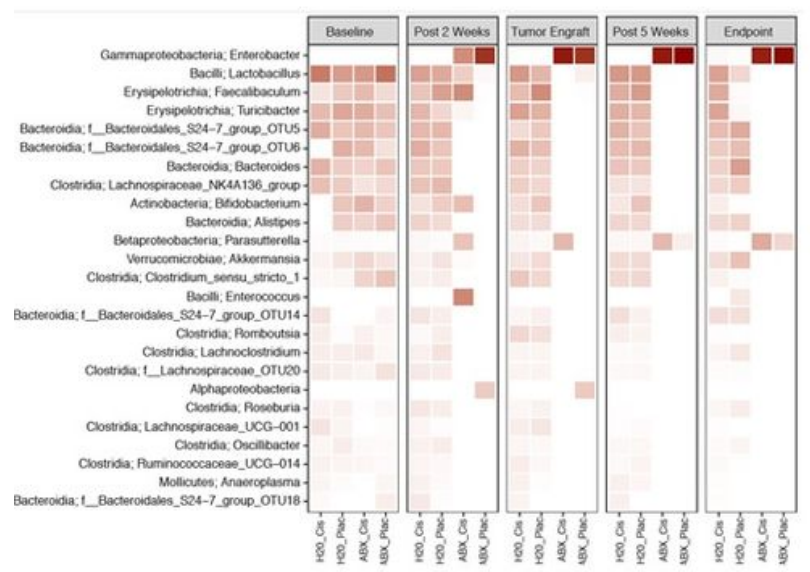

B.

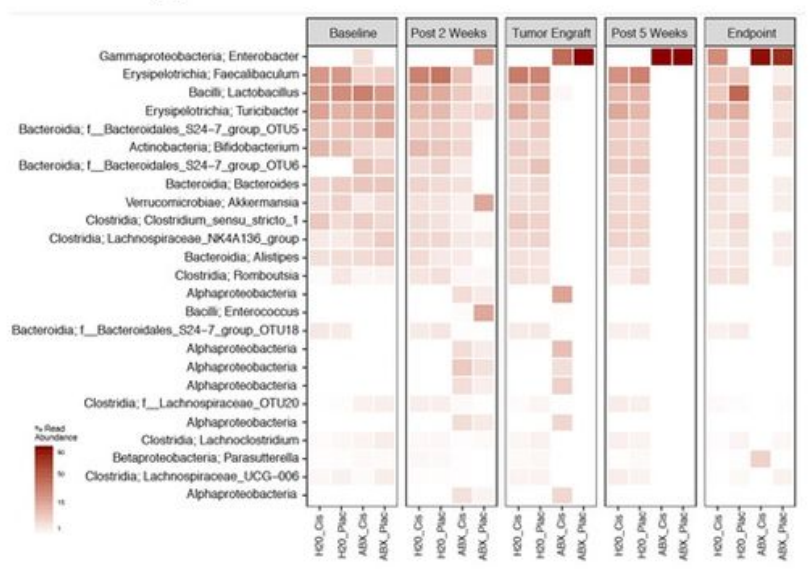

Figure 3 
$A B X$ therapy results in microbial alterations over time in the gut microbiome of C57BL/ 6 mice. Specific strains of bacteria are altered in the ID8 (A) and ID8 VEGF (B) cohorts of C57 BL/6 mice following antibiotic treatment over time. $\mathrm{n}=8$ mice per group

A.

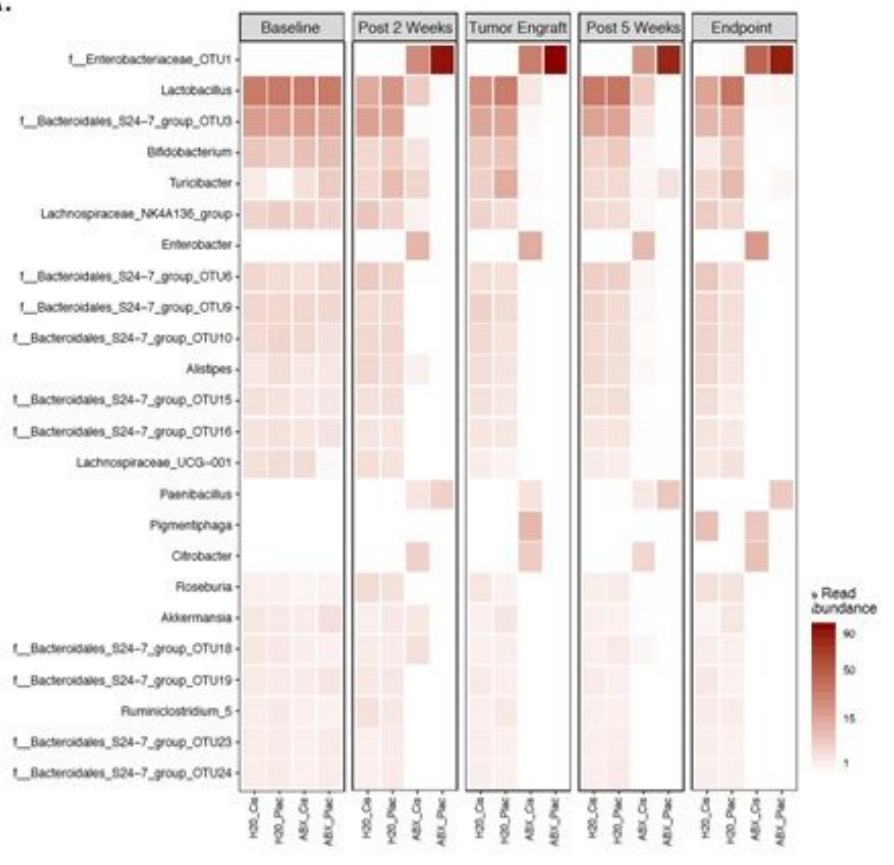

B.

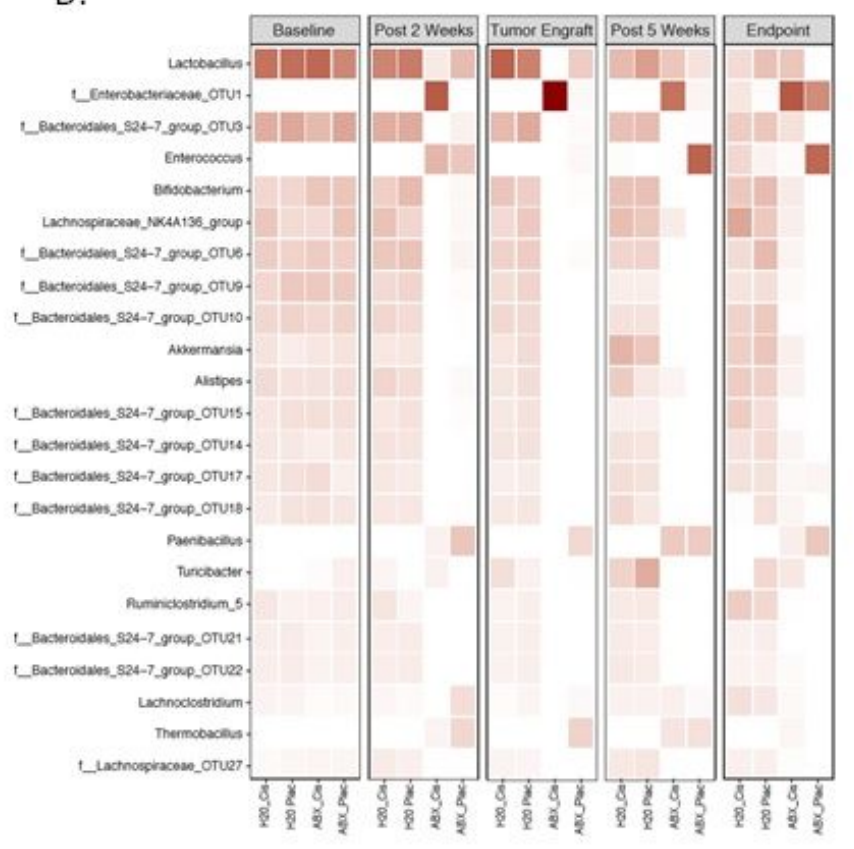

Figure 4

$A B X$ therapy results in microbial alterations over time in the gut microbiome of NSG mice. Specific strains of bacteria are altered in the ID8 (A) and ID8 VEGF (B) cohorts of NSG mice following antibiotic treatment over time. $n=8$ mice per group 

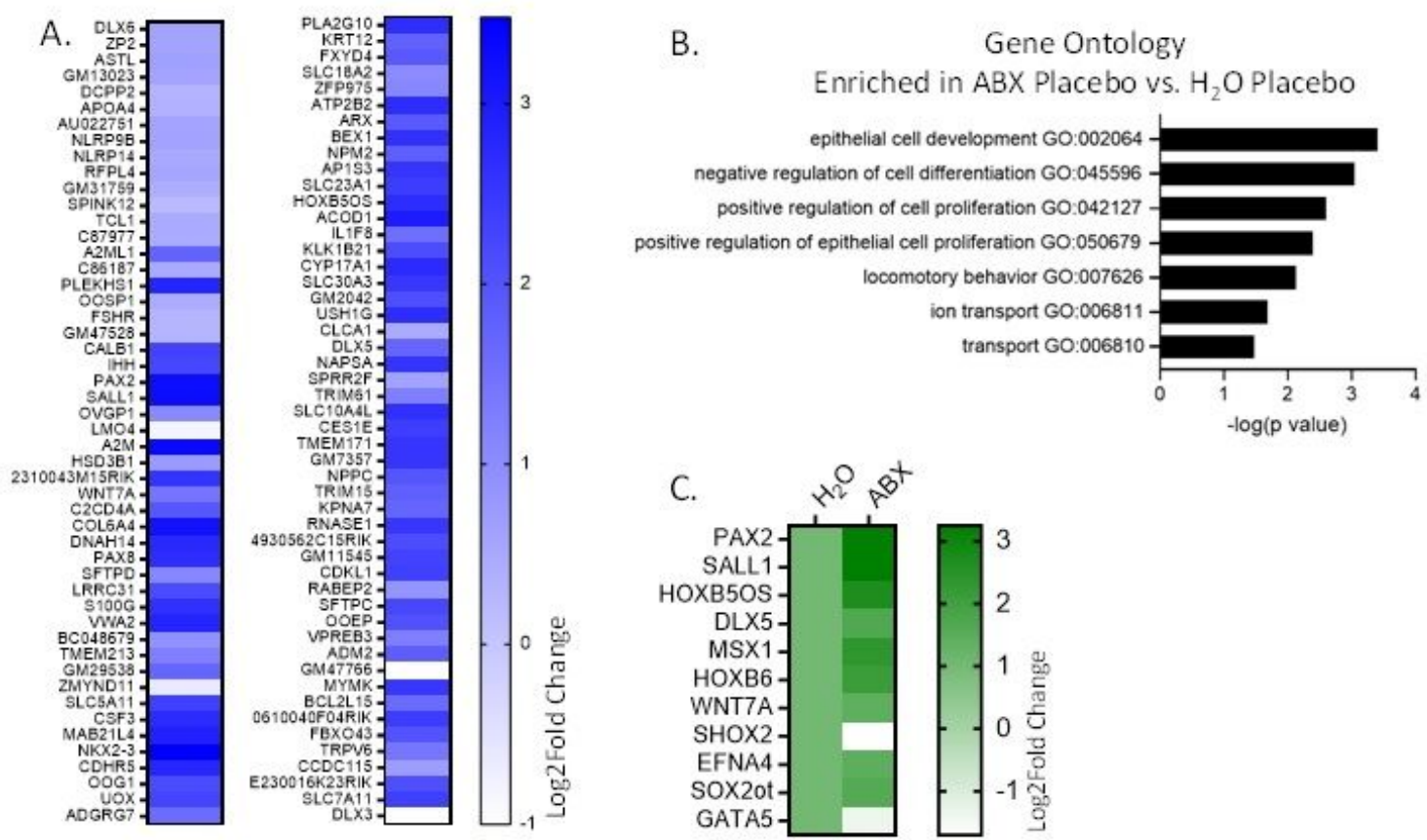

D.

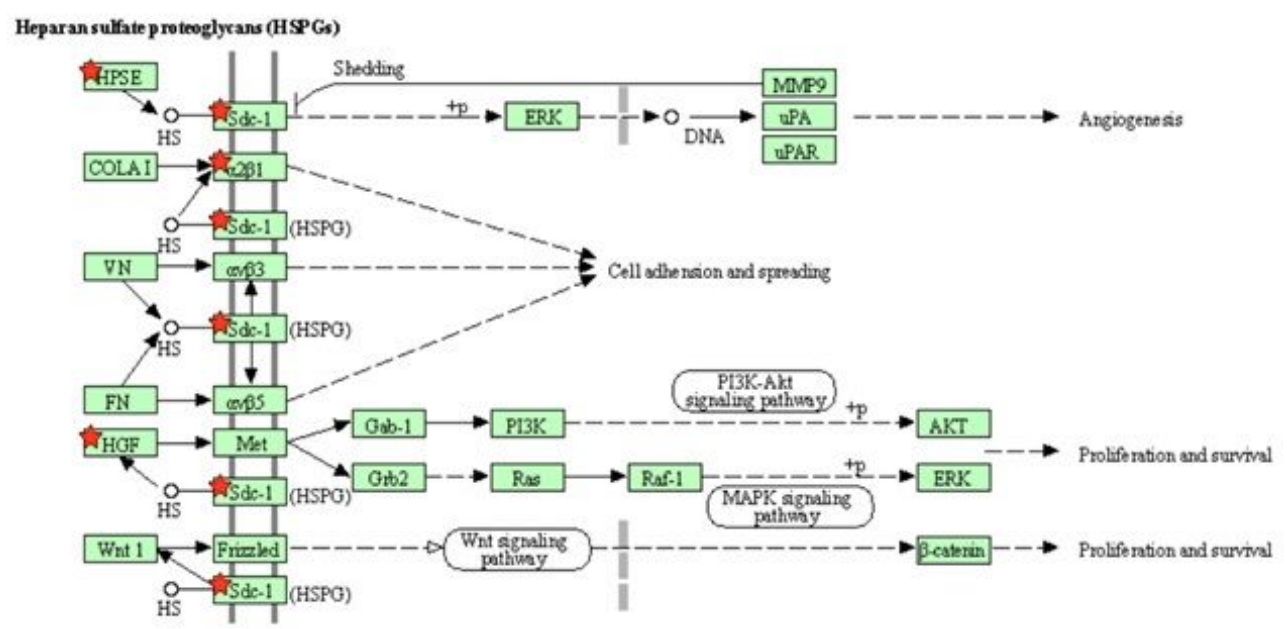

\section{Figure 5}

RNAseq of NSG ID8 tumors at endpoint demonstrates differentially expressed genes between treatment groups. (A) Top 100 differentially expressed genes (DEGs) ordered by p-value in the ABX Placebo group over the H2O Placebo group. (B) Top significant $\mathrm{GO}$ terms enriched in the ABX Placebo group over $\mathrm{H} 2 \mathrm{O}$ Placebo group. (C) Stem cell genes enriched in the ABX Placebo group over the $\mathrm{H} 2 \mathrm{O}$ Placebo group ordered by p-value. (D) KEGG pathways enriched in ABX Placebo group over $\mathrm{H} 2 \mathrm{O}$ Placebo group, enriched genes with a $\mathrm{p}$ value $<0.01$ are denoted by red stars. 
A.

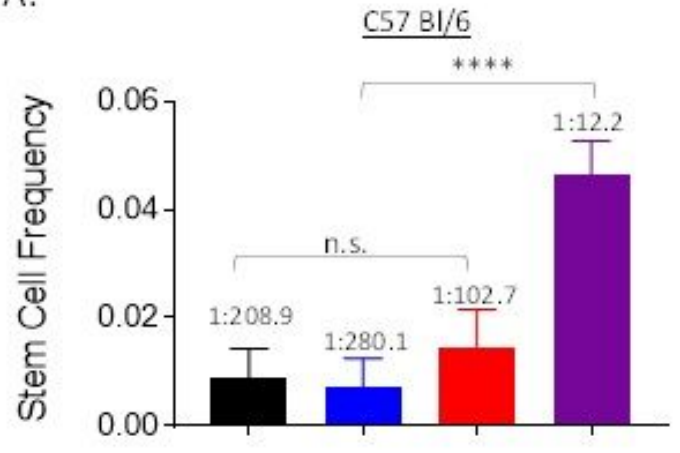

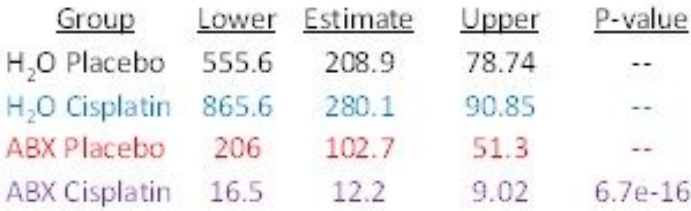

B.

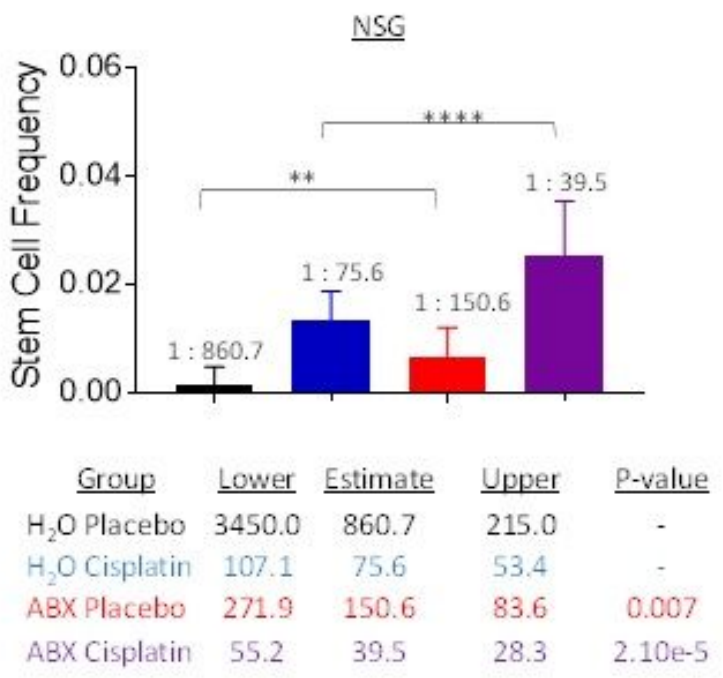

\section{Figure 6}

Tumor sphere initiation frequency is increased in $A B X$ treated mice and further increased upon cisplatin therapy. ID8 tumor cells isolated from $A B X$ treated mice had significantly increased tumor sphere initiation frequency in both $B L / 6$

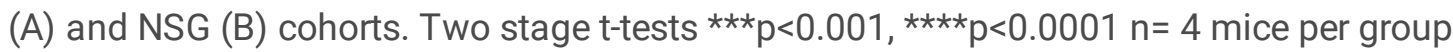

\section{Supplementary Files}

This is a list of supplementary files associated with this preprint. Click to download.

- supplement4.docx 